\title{
Peculiarities of neutron irradiation influence on GaP light-emitting structures
}

\author{
P. Litovchenko, A. Litovchenko, O. Konoreva, V. Opilat ${ }^{*}$, M. Pinkovska, V. Tartachnyk \\ Institute for Nuclear Researches, NAS of Ukraine, 47, prospect Nauky, 03028 Kyiv, Ukraine \\ *M. Drahomanov National Pedagogical University, 9, Pirohova str., 03023 Kyiv, Ukraine \\ Corresponding author: phone+38(044)-525-39-97, +38(044)-525-37-49; e-mail: okskon@meta.ua
}

\begin{abstract}
GaP LEDs irradiated by reactor neutrons were studied by optical and electrical methods. The observed emission intensity degradation is related with two factors: 1) radiation fields that destroy bond excitons and 2) decrease in the free charge carrier concentration, which is caused by their capture by radiation defects. Study of currentvoltage characteristics at $77 \mathrm{~K}$ by highly precession methods has revealed the appearance of N-shaped negative differential region caused by carrier tunneling onto deep levels in quantum wells, which might exist in initial and irradiated $p-n$ structures. In some cases, improvement of current-voltage characteristics after neutron irradiation is observed. An assumption is made about the radiation-stimulated origin of this effect.
\end{abstract}

Keywords: GaP LEDs, irradiation, emission degradation, negative resistance, oscillation.

Manuscript received 15.04.09; accepted for publication 14.05.09; published online 29.05.09.

\section{Introduction}

Light emitting diodes (LEDs) are widely used in computing systems, contemporary high frequency intercommunication, monitoring, diagnostics domestic devices due to small size, simplicity and low cost. Despite technology advantages, the quantum yield of diodes is not stable enough and decreases during diode operation $[1,2]$. The radiation stability increase is the issue of the day for all LED types. Later, the influence of irradiation on GaP LEDs was studied in [3-5]. Thermal stability of radiation defects in GaP LEDs is not clear up to date. The radiation trap parameters and the thermal stability range of radiation induced defects can be obtained by analysis of electrical characteristics of LEDs after irradiation. Some features of current-voltage characteristics for irradiated LEDs were presented in our previous study [6]. In the abovementioned paper, the electrical and optical properties of $\mathrm{CZ} \mathrm{GaP} p$ - $n$ junctions used as substrates for LEDs irradiated by reactor neutrons were studied and compared with the electronirradiated ones.

\section{Experiment and results}

The studied GaP light diodes were processed by double liquid epitaxy. Current-voltage characteristics $(C-V)$ of $p-n$ structures were measured at nitrogen and room temperatures. The thin structure of $C-V$ of $p-n$ junction has been measured by special technique that allows to change precisely current or voltage steps, which makes it possible to do measurements nearly momentary. Samples were characterized by DLTS and emission spectra measurements. The structures were irradiated by reactor neutrons at $50{ }^{\circ} \mathrm{C}$ and electrons $(E=2 \mathrm{MeV})$.

Introduction of the radiation defects into GaP LEDs causes decrease of the emission intensity $I$ (Fig. 1), and can be expressed as $I=I_{0} \exp (-k \Phi)$. The average value of the damage coefficient for reactor neutrons $(\bar{E}=1 \mathrm{MeV})$ obtained from the abovementioned expression is $k_{n 1}=1.18 \cdot 10^{-13} \mathrm{~cm}^{2}$ for the fluence range $\Phi=(0 \ldots 9) \cdot 10^{12} \mathrm{~cm}^{-2}$, and $k_{n 2}=0.59 \cdot 10^{-13} \mathrm{~cm}^{2}-$ for $\Phi=$ $9 \cdot 10^{12}-10^{14} \mathrm{~cm}^{-2}$. The average value of the damage coefficient $k_{e}$ is $(9.2 \ldots 4.5) \cdot 10^{-16} \mathrm{~cm}^{2}$ for electron fluences $(0 \ldots 2) \cdot 10^{15} \mathrm{~cm}^{-2}$ and $2 \cdot 10^{15}-4 \cdot 10^{16} \mathrm{~cm}^{-2}$, respectively. The comparison of neutron and electron cases gives for the initial irradiation fluences the ratio $k_{n 1} / k_{e 1} \approx 10^{2}$. Thus, the reactor neutron radiation damage is much higher than that of the electron one and can be explained by the complex defect formation. Therefore, thermal stability of the neutron induced radiation defects is high. As seen from Fig. 2, the second stage of annealing the radiation defects, i.e., annealing of the disorder regions, occurs at $T>500{ }^{\circ} \mathrm{C}$, while point defects $\left(\mathrm{V}_{\mathrm{Ga}}\right.$ and $\mathrm{V}_{\mathrm{P}}$, induced by reactor gamma-irradiation) are annealled at 100 to $200{ }^{\circ} \mathrm{C}$. 


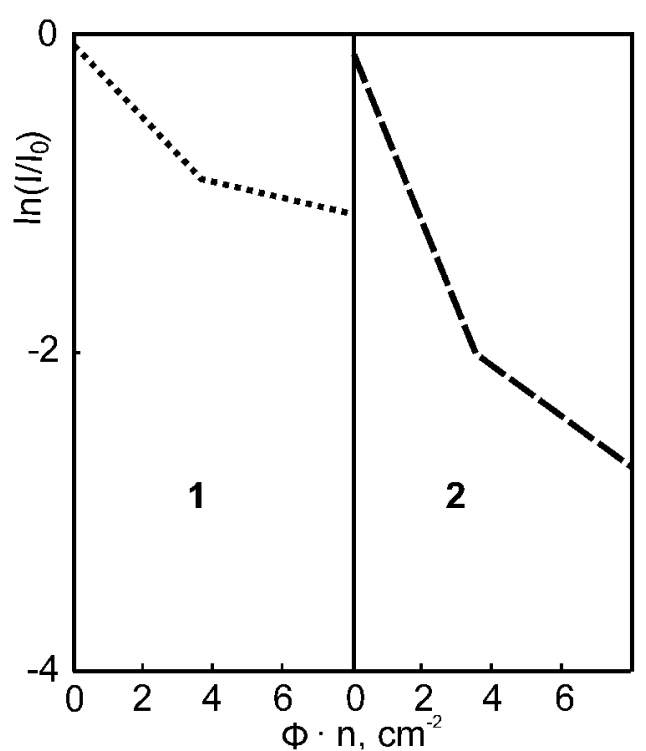

Fig. 1. Intensity emission drop as the function of radiation with: $\quad 1-$ neutrons, $\quad n=11.8 \cdot 10^{12} \mathrm{~cm}^{-2} ; 2-$ electrons, $n=2.6 \cdot 10^{14} \mathrm{~cm}^{-2}$.

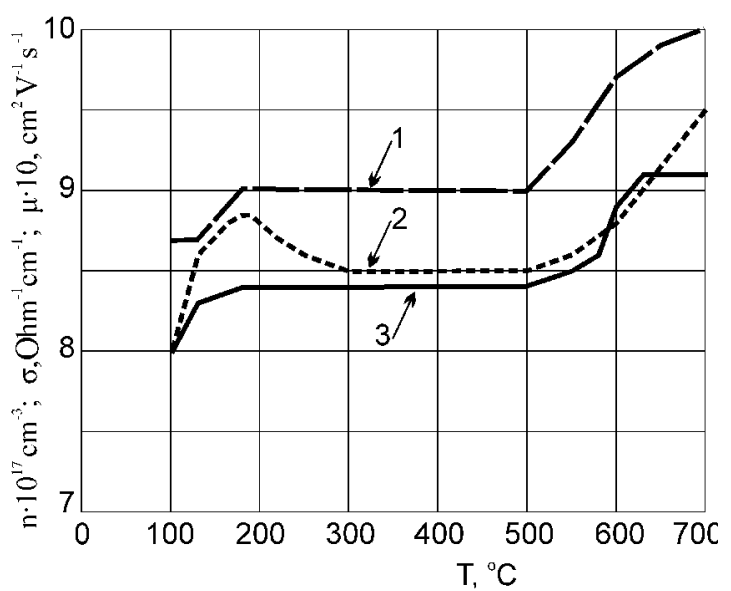

Fig. 2. Annealing of the concentration $n(1)$, conductivity $\sigma(2)$ and mobility (3) of GaP LEDs irradiated with neutrons $\Phi=10^{16} \mathrm{~cm}^{-2}$

After electron irradiation, we observed five deep levels in $\mathrm{GaP}$ forbidden gap appearing with the introduction rate $6.2 \mathrm{~cm}^{-1}$ measured by DLTS technique. This value agrees well with the carrier removal rate.

Thus, each induced defect captures one free carrier. It can be suggested that radiation defects are monovalent. The concentration of deep levels with the energy $E=0.79 \mathrm{eV}$ increases.

In neutron-irradiated samples $\left(\Phi=3 \cdot 10^{14} \mathrm{~cm}^{-2}\right)$ only $0.2-0.7 \mathrm{eV}$ wide band appears with the mean concentration of induced defects $5 \cdot 10^{15} \mathrm{~cm}^{-3}$. After neutron irradiation, the concentration of deep levels with the energy $E=0.79 \mathrm{eV}$ also presented in $\mathrm{GaP}$ forbidden gap of non-irradiated diodes increases. The main changes in DLTS spectra of neutron irradiated GaP diodes are observed after $400{ }^{\circ} \mathrm{C}$ annealing. The concentration of traps with the energy $E=0.2-0.7 \mathrm{eV}$ decreases down to $3 \cdot 10^{14} \mathrm{~cm}^{-3}$ and new traps with the concentration $6 \cdot 10^{15} \mathrm{~cm}^{-3}$ and energy $E=0.71 \mathrm{eV}$ appear.

The main peculiarities of the GaP LEDs are in appearance of current instabilities in the region of negative resistance in $C-V$ characteristics at $100-77 \mathrm{~K}$. These oscillations were observed earlier [6] and related with filling and depletion of deep levels.

Detailed analysis of $C$ - $V$ characteristics measured in voltage-generator mode at the nitrogen temperature has also shown N-type irregularities both in forward and reverse characteristics. In some samples, especially in the irradiated ones, these characteristics might be divided into separate curves, which follow one by one. Neutron irradiation leads to appearance of a thin structure in the N-type region of direct $C-V$ characteristics and the current oscillation amplitude increases (Fig. 3).

The similar results are observed for the reverse $C-V$ curve, and nature of the current oscillations differs after annealing (Fig. 4). The $I_{\text {rev }}(V)$ curve demonstrates the deep minimal current. This value decreases with repetition of these measurements (Fig. 5). The N-shaped anomalies in both parts of current-voltage characteristics can be explained by current tunneling into large-scale defects of quantum well-type [7]. They are present in initial samples and irradiation may influence on it. The electric field $10^{6} \mathrm{~V} / \mathrm{cm}$ has been applied to the $p$ - $n$ transition region. This value is enough to stimulate tunneling currents under conditions when the level of the defect quantum well as caused by the band shift is opposite as compared to that of the conductive/valence band edge. The change of the minimal current in reverse $C-V$ characteristics after the repeating measurements (Fig. 5) confirmed this hypothesis, each followed current-voltage measure causes changes in the level filling of quantum wells.

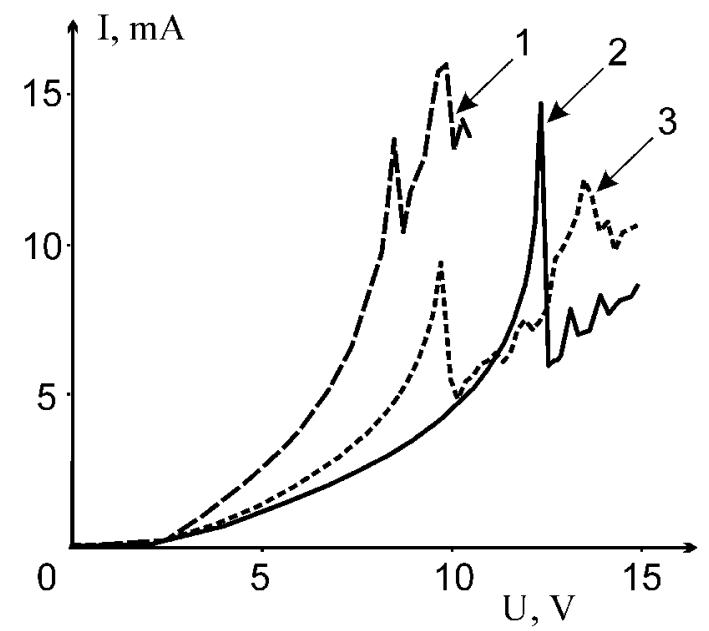

Fig. 3. Current-voltage characteristics of GaP, $\mathrm{Zn}: \mathrm{O}$ measured in voltage-generator mode at $77 \mathrm{~K}: 1-$ initial, $2-\Phi=$ $4.5 \cdot 10^{16}$ neutrons $/ \mathrm{cm}^{2}, 3-$ annealled $600{ }^{\circ} \mathrm{C}$. 


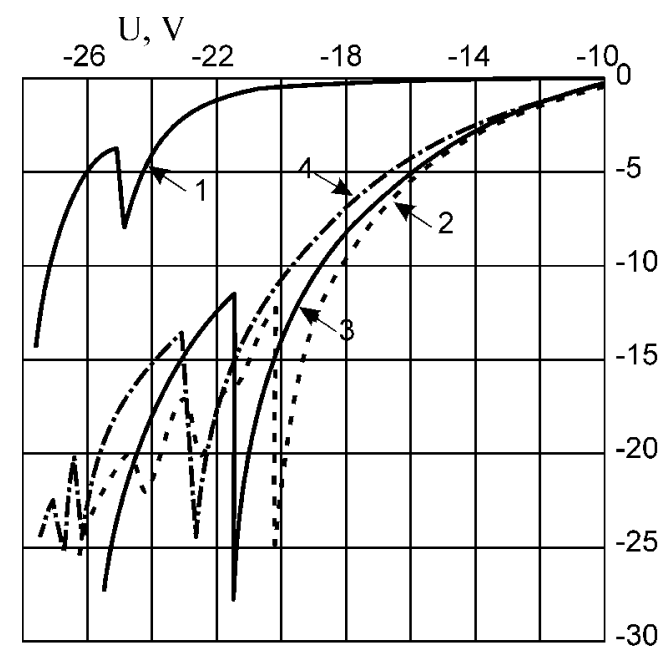

$\mathrm{I}, \mathrm{mA}$

Fig. 4. Reverse current-voltage characteristics of $\mathrm{GaP}, \mathrm{Zn}: \mathrm{O}$ annealled at temperatures: 1 - initial, $2-80,3-110,4-$ $130{ }^{\circ} \mathrm{C}$.

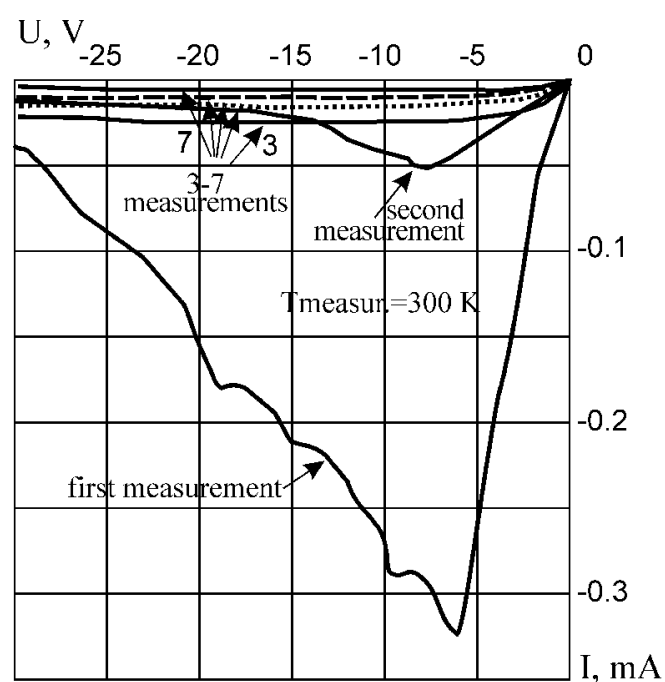

Fig. 5. Reverse current-voltage characteristics of GaP LEDs when repeating the measurements.

Improvement of electrical characteristics obvious in neutron-irradiated $\left(\Phi \leq 10^{14} \mathrm{~cm}^{-2}\right) \mathrm{GaP}$ LEDs at room temperatures is expressed by direct current increase, while reverse current and oscillation amplitudes in $\mathrm{N}$ and S-shaped regions decreases. The differential resistance decreases at low direct currents and biases, and the point of intersection of forward $C-V$ characteristics for non-irradiated and irradiated diodes goes down with the neutron dose increase (Fig. 6).

\section{Discussion}

The irradiation introduces deep levels into the GaP forbidden gap, which may capture the majority current carriers. The Fermi level shifts to the middle of forbidden gap and reaches the value $E_{g} / 2$ with the neutron fluence increase. But conversion of the conductivity type is not observed even for high fluences.

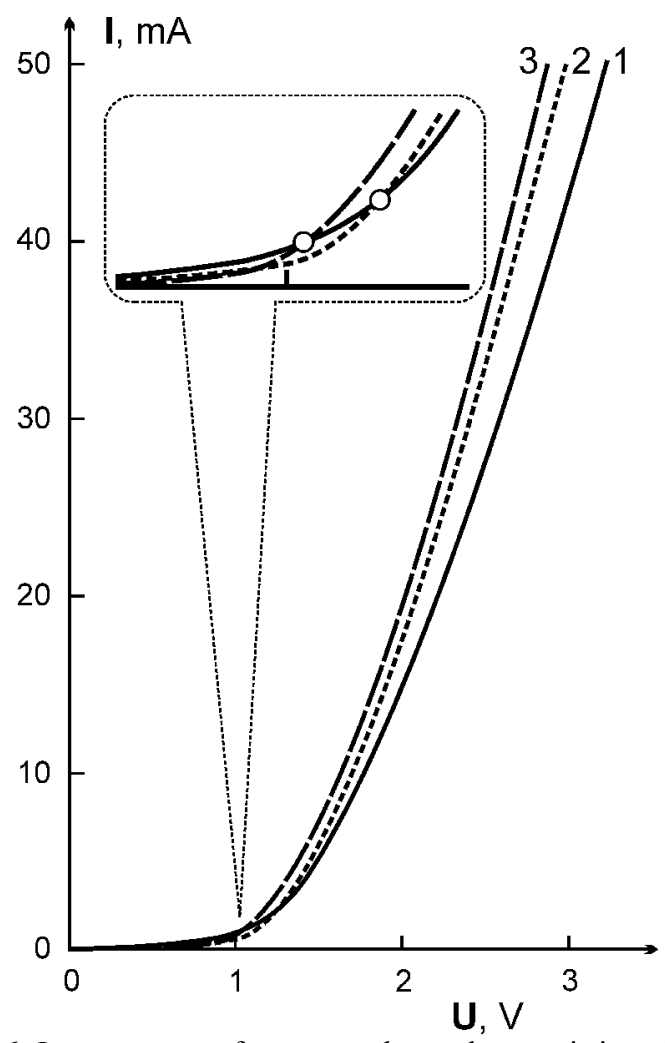

Fig. 6. Improvement of current-voltage characteristics of $\mathrm{GaP}$ LEDs after neutron irradiation: 1 - initial, 2 - first fluence, 3 second fluence.

The emission characteristics are very sensitive to radiation, so the effect is related with the annihilation of the bound excitons. The emission intensity decreases at low neutron fluences. The changes in electrical characteristics are not constant. This emission degradation depends exponentially on the fluence. Radiation influences on the radiative centers and destroys excitons. The ratio of radiation damage coefficients for neutrons and electrons at the beginning of irradiation $\left(\sim 10^{2}\right)$ confirms this statement. The sharp bend in Fig. 1 divides the experimental curve by two parts: the region of low fluences, where excitons are destroyed by radiation, and the high fluence region, where, apparently, this effect is accompanied by the decrease of the majority carriers' concentration in GaP.

The emission intensity degradation was evaluated using DLTS measurement results. The comparison of annealing temperatures for traps obtained by DLTS measurements as well as annealing stages of $\mathrm{V}_{\mathrm{Ga}}$ and $\mathrm{V}_{\mathrm{P}}$ in $\mathrm{GaP}$ shows that the level $E=0.6 \mathrm{eV}$ belongs to $\mathrm{V}_{\mathrm{Ga}}$. Other levels are related with $\mathrm{V}_{\mathrm{Ga}}$ complex defects. The wide band $0.2-0.7 \mathrm{eV}$ in DLTS spectra is the result of superposition of nearly located levels in neutron irradiated diodes. We suppose that this band is formed by defects of disordered regions. The increase in the concentration of the level $E=0.71 \mathrm{eV}$ at high annealing temperatures is the result of the beginning of complex defect decay. The disordered regions dissociate for 
components such as complexes of radiation defect and impurity/defect associations, for example.

Improvement of diode electrical characteristics after neutron irradiation has to be studied. It is possible to assume only that radiation-stimulated transformation occurs [7]. The impurity diffusion in the depletion region becomes stronger under high ionization levels and under sufficient electrical $p$ - $n$ junction field.

\section{Conclusion}

1. Our study of emission characteristics of GaP LEDs irradiated by reactor neutrons and electrons has confirmed that destructive influence of neutrons is higher than electrons and is related with complex defect formation. Emission intensity degradation of neutron-irradiated diodes at medium fluences is related with two factors: the radiation that destroys the bound excitons and the decrease of free carriers due to their capture by radiation defects.

2. From DLTS spectra parameters of same traps with the energies $E=0.25,0.29,0.33,0.55$, and $0.60 \mathrm{eV}$ were revealed after electron irradiation. The mean trap incorporation rate is $6.2 \mathrm{~cm}^{-1}$. In neutronirradiated samples $\left(\Phi=3 \cdot 10^{14} \mathrm{~cm}^{-2}\right)$, the only 0.2 $0.7 \mathrm{eV}$ wide band appears with the mean concentration of induced defects $5 \cdot 10^{15} \mathrm{~cm}^{-3}$.

3. The region of N-shaped NDR was observed at $77 \mathrm{~K}$ and caused by the carrier tunneling in deep levels of quantum wells, which are in non-irradiated and irradiated $p$ - $n$ structures.

4. Improvement of $\mathrm{GaP}$ diode current-voltage characteristics after neutron irradiation is observed. An assumption is made about the radiationstimulated nature of this effect.

\section{References}

1. A. Berg, P. Din. Light Emitting Diodes. Mir Publ., Moscow, 1979 (in Russian).

2. R.I. Hartman, B. Schwartz, M. Kuhn, Degradation and passivation of GaP light-emitting diodes // Appl. Phys. Lett. 18, p. 304-306 (1971).

3. A. Polity, T.N. Abgargan, R. Krause-Rohberg, Defects in electron irradiated $\mathrm{GaP}$ studied by positron lifetime spectroscopy // J. Appl. Phys. A 60, p. 541-544 (1995).

4. T. Kawakubo and M. Okada, Electrical and optical properties of neutron-irradiated $\mathrm{GaP}$ crystals // J. Appl. Phys. A 67, p. 3111-3114 (1990).

5. A.H. Johnson, B.G. Rax, L.E. Selva, and C.E. Barnes, Proton degradation of light-emitting diodes // IEEE Trans. Nucl. Sci. 46, p. 1781-1789 (1999).

6. P. Litovchenko, D. Bisello, A. Litovchenko, S. Kanevskyj, V. Opilat, M. Pinkovska, V. Tartachnyk, R. Rando, P. Giubilato, Some features of current-voltage characteristics of irradiated GaP light diodes // Nucl. Instrum. Meth. A 552, p. 93-97 (2005).

7. V.E. Kudrishov, K.G. Zolin, A.N. Turkin, A.E. Yunovich, A.N. Kovalev, F.I. Maniakhin, Tunnelling in light-emitting diodes based on InGaN/AlGaN/GaN heterostructures with quantum wells // Fizika $i$ tekhnika poluprovodnikov 31, p. 1304-1309 (1997) (in Russian).

8. O.Yu. Borkovskaya, N.L. Dmytruk, V.G. Lytovhenko, O.N. Mishchuk, On the model of the effect of radiation-stimulated ordering in semiconductors $\mathrm{A}^{3} \mathrm{~B}^{5}$ // Fizika i tekhnika poluprovodnikov 23, No. 2, p. 207-212 (1989) (in Russian). 